\title{
EFFECT OF THE ORDER ON THE STRUCTURAL AND MAGNETIC PROPERTIES OF Fe-X (X=Al, Si) ALLOYS WITH B2 STRUCTURE
}

\author{
J. DENISZCZYK \\ Institute of Physics and Chemistry of Metals, Silesian University \\ Bankowa 12, 40-007 Katowice, Poland
}

\begin{abstract}
Previous $a b$ initio super-cell calculations of the magnetic properties of the ordered $\mathrm{Fe}_{1-x} \mathrm{Al}_{x}$ alloy in the $\mathrm{B} 2$ structure are extended to include the disordered state of the alloy and completed with the calculations for ordered and disordered $\mathrm{Fe}_{1-x} \mathrm{Si}_{x}$ alloys. The configurations of the Fe atoms in the anti-site (AS) positions of $\mathrm{Fe}-\mathrm{X}$ super-cell with the antiferromagnetic order were found. The ground state of $\mathrm{Fe}_{1-x} \mathrm{Al}_{x}$ with $x \simeq 0.3$ shows the antiferromagnetic order of the $\mathrm{Fe}-\mathrm{AS}$ magnetic moments. The concentration dependence of the equilibrium lattice parameter and magnetic moment of $\mathrm{Fe}_{1-x} \mathrm{X}_{x}$ in the ordered and disordered state differ qualitatively.
\end{abstract}

PACS numbers: 71.15.La, 71.15.Fv, 71.20.Lp, 75.50.Bb, 75.30.-m

The ordered $\mathrm{Fe}-\mathrm{Al}$ alloys with the B2-type structure display a complicated structural and magnetic behavior. In the concentration range of $x=0.25-0.5$ the $\mathrm{B} 2 \leftrightarrow \mathrm{DO}_{3}$ transitions are observed and the magnetic phase diagram for the $\mathrm{Fe}_{1-x} \mathrm{Al}_{x}$ shows unusual magnetic features [1, 2]. The magnetic state of $\mathrm{Fe}_{1-x} \mathrm{Al}_{x}$ with $x \simeq 0.3$ was found paramagnetic for $T \geq 400 \mathrm{~K}$, ferromagnetic in temperatures $170 \mathrm{~K} \leq T \leq 400 \mathrm{~K}$, paramagnetic for $92 \mathrm{~K} \leq T \leq 170 \mathrm{~K}$ and goes to the spin-glass behavior below $92 \mathrm{~K}$. Neutron-spectroscopy studies [3] prove that the magnetic moment of iron is strictly related to the number of $\mathrm{Al}$ in the first coordination sphere. The magnetic moment of Fe atoms with only Fe near neighbors is equal to $\simeq 2.2 \mu_{\mathrm{B}}$ and falls down to $\simeq 1.5 \mu_{\mathrm{B}}$ when $4 \mathrm{Al}$ atoms are on the first coordination sphere. Arrot et al. and Shiga et al. [5] postulated the antiferromagnetic coupling between the iron magnetic moments to be responsible for the unusual magnetic behavior of the $\mathrm{Fe}-\mathrm{Al}$ ordered alloys. The electronic structure calculations revealed that the $\mathrm{FeAl}$ equiatomic compound has the metastable antiferromagnetic state with energy close to the ferromagnetic state [6]. The antiferromagnetic alignment of $\mathrm{Fe}$ magnetic moments in $\mathrm{Fe}-\mathrm{Al}$ finite clusters was also found by Elzain et al. [7]. Despite the fact that the alloys were studied widely with the use of both experimental and theoretical methods the microscopic mechanism of the anomalous structural and magnetic behavior is still not known. 
The aim of the investigations is to explain the influence of the structural order on the structural and magnetic properties of $\mathrm{Fe}-\mathrm{Al}$ and $\mathrm{Fe}-\mathrm{Si}$ alloys. The previously presented results [8] are extended to wider concentration range of $\mathrm{Al}$ in $\mathrm{Fe}_{1-x} \mathrm{Al}_{x}(x=0.0 \div 0.5)$. In order to study the effect of the atomic order the calculations for the disordered state were performed. For the purpose of comparison the results for the ordered and disordered $\mathrm{Fe}_{1-x} \mathrm{Si}_{x}$ alloys are also reported.

For the case of the ordered alloys the super-cell approach was applied and the electronic structure calculations were performed with the use of the tight-binding linear muffin tin orbital (TB-LMTO) method in the atomic sphere approximation (ASA) [9]. The calculations were scalar-relativistic (the spin-orbit coupling is neglected). The local spin density exchange-correlation potential [10] with gradient corrections [11] was used. The details of data preparations are presented in [8]. The calculations for the disordered alloys were performed within the Korringa-KohnRostoker formalism with the use of coherent potential approximation approach developed for binary alloys by Tatarchenko et al. [12]. The crystal potential was taken within the ASA, and the calculations were non-relativistic.

The results of the super-cell calculations are displayed in Figs. 1 and 2. Both figures present the variation of the lattice constants and average magnetic moments with metalloid concentrations in $\mathrm{Fe}_{1-x} \mathrm{X}_{x}$. Figure 1 shows the results of the super-cell approach.
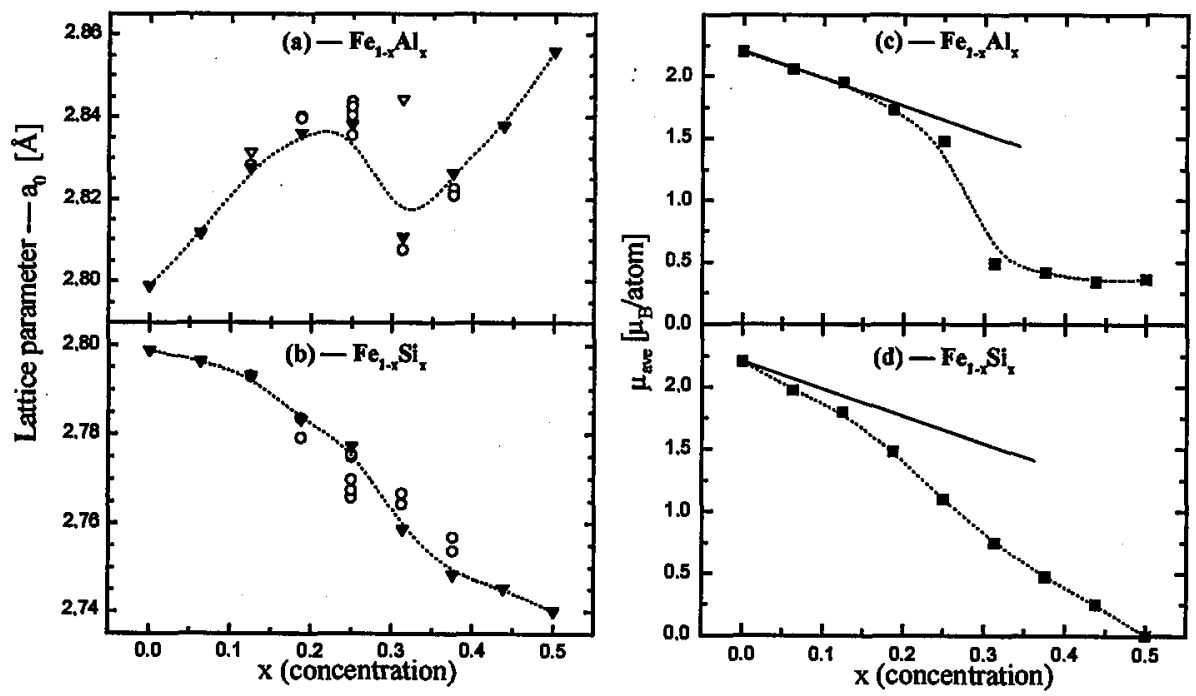

Fig. 1. Equilibrium lattice parameter $a_{0}$ (left panel) and the average magnetization per atom $\mu_{\text {ave }}$ (right panel) of the ordered $\mathrm{Fe}_{1-x} \mathrm{Al}_{x}$ and $\mathrm{Fe}_{1-x} \mathrm{Si}_{x}$. For a given cencentration the solid and open symbols in (a) and (b) represent the Fe-AS atoms' configurations with the minimal and higher total energies, respectively. Solid symbols in (a) and (c) represent the $\mathrm{Fe}-\mathrm{AS}$ atom configurations with the minimum total energy for a given concentration. Solid lines in (c) and (d) display the simple dilution law. 
The super-cell calculations for $\mathrm{Fe}_{1-x} \mathrm{Al}_{x}$ give the variation of the lattice constant with the aluminum content which qualitatively reproduce the experimental results obtained for $\mathrm{Fe}-\mathrm{Al}$ alloys quenched from $1000^{\circ} \mathrm{C}$ [5]. The dependence of the average magnetic moment on $\mathrm{Al}$ concentration also follows the measured magnetization of the ordered $\mathrm{Fe}-\mathrm{Al}$ alloys [13]. The changes of $a_{0}$ shown in Fig. 1a may be related to that of the average magnetic moment $\mu_{\text {ave }}$ by the empirical relation similar to that proposed in [5]. Based on the results displayed in Figs. 1a and c the fitting procedure gives the coefficient of the $a_{0}\left(x, \mu_{\text {ave }}\right)$ versus $\mu_{\text {ave }}$ dependence equal $0.0477 \AA / \mu_{\mathrm{B}}$, close to that obtained by Shiga $\left(0.0355 \AA / \mu_{\mathrm{B}}\right)[5]$.

A completely different behavior shows the lattice parameter calculated for the ordered $\mathrm{Fe}_{1-x} \mathrm{Si}_{x}$ alloys Fig. $1 \mathrm{~b}$. In this alloys $a_{0}$ decreases with increasing $\mathrm{Si}$ contents. The effect is analyzed and the results will by published elsewhere. According to the results obtained for ordered $\mathrm{Fe}_{1-x} \mathrm{Si}_{x}$ (Fig. 1d) the average magnetic moment for small concentrations of Si decreases faster than the simple dilution law predicts but the decrease is constant without any abrupt changes like in $\mathrm{Fe}_{1-x} \mathrm{Al}_{x}$.

The calculations performed for the disordered $\mathrm{Fe}_{1-x} \mathrm{X}_{x}$ alloys are summarized in Figs. 2a,b. The calculated increase in the lattice parameters can be explained as due to the increasing number of larger volume metalloid atoms. The magnetic moment of disordered $\mathrm{Fe}_{1-x} \mathrm{X}_{x}$ decreases faster than it could be expected from the dilution law because the $\mathrm{Al}(\mathrm{Si})$ atoms have small negative magnetic moments.

The most interesting result of the super-cell approach was the finding of the antiferromagnetic coupling between the magnetic moments on the $\mathrm{Fe}$ atoms at anti-site positions ( $\mathrm{Fe}-\mathrm{AS}$ ). Figure 3 shows some of the calculated configurations with anti-parallel alignment of $\mathrm{Fe}-\mathrm{AS}$ magnetic moments. The antiferromagnetically ordered $\mathrm{Fe}-\mathrm{AS}$ moments are almost of the same magnitude. The construction of the super-cell and the translational invariance cause that the sites of the Fe-sublattice remain equivalent which prohibits any magnetic disorder. Therefore the magnetic moments of $\mathrm{Fe}-\mathrm{AS}$ atoms are immersed in the uniform, magnetically ordered iron sublattice. Calculations of different configurations of the $\mathrm{Fe}-\mathrm{AS}$ atoms support the hypothesis that the interaction responsible for such antiferrimagnetism is the RKKY-type interaction between the magnetic moments of next
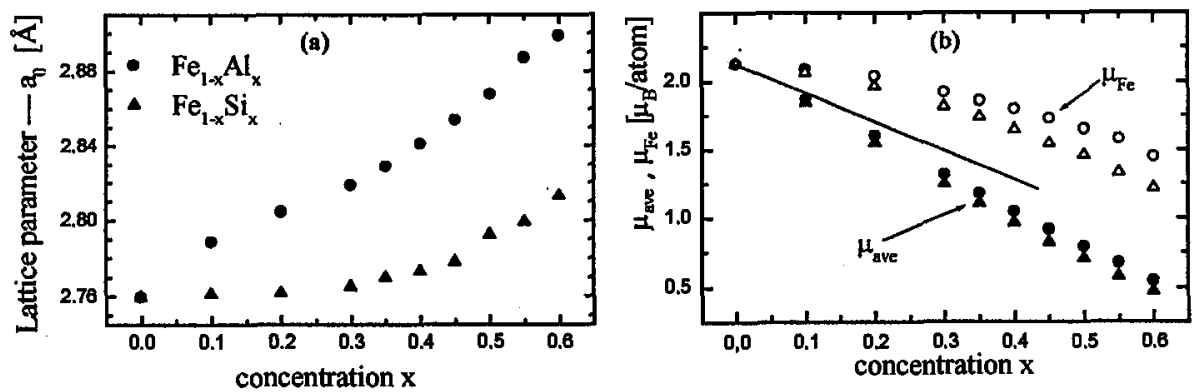

Fig. 2. Lattice parameter $a_{0}(\mathrm{a})$ and the iron $\left(\mu_{\mathrm{Fe}}\right)$ and the average $\left(\mu_{\mathrm{ave}}\right)$ magnetic moments per atom (b) calculated for the disordered $\mathrm{Fe}_{1-x} \mathrm{Al}_{x}$ and $\mathrm{Fe}_{1-x} \mathrm{Si}_{x}$. Solid line in (b) shows the simple dilution law. 
(a)

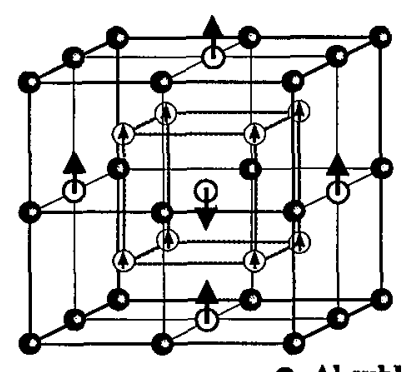

(b)

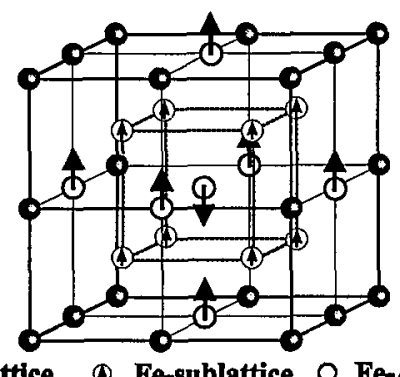

(c)

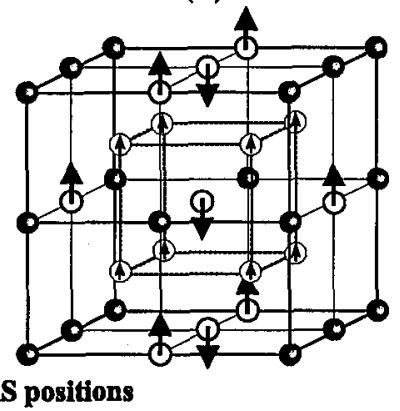

Fig. 3. Configurations of $\mathrm{Fe}_{1_{-x}} \mathrm{Al}_{x}$ super-cell with antiferromagnetic alignment of Fe-AS magnetic moments. Configuration (a) belongs to a concentration of $x=0.3125$ while (b) and (c) to $x=0.25$.

neighboring $\mathrm{Fe}-\mathrm{AS}$ atoms. The effect occurs in both the investigated alloys. The difference is that in the case of $\mathrm{Fe}_{1-x} \mathrm{Al}_{x}$ the low-magnetic (LM) solutions are close in energy to the high-magnetic (HM) ones. In the case of $x \simeq 0.3$ such LM solution is the ground state of the super-cell. In $\mathrm{Fe}_{1-x} \mathrm{Si}_{x}$ the LM solutions with anti-parallel $\mathrm{Fe}-\mathrm{AS}$ magnetic moments always lie above the HM ones and never occur as a ground state. In conclusions it can be stated that a certain unusual magnetic and structural behavior can be explained as an effect of the atomic order. The result of antiferromagnetic coupling between the $\mathrm{Fe}-\mathrm{AS}$ magnetic moments is surely related to the geometry of the investigated super-cell. The problem is under consideration. The presented results should be completed by the calculations using different methods allowing for the fixed moment approach. Such calculations within the full potential LAPW method are in progress.

This work was supported in part by the Committee for Scientific Research under the contract No. 2 P03B 12914.

\section{References}

[1] R.D. Shull, H. Okamoto, P.A. Beck, Solid State Commun. 20, 863 (1976).

[2] K. Motoya, S.M. Shapiro, Y. Muraoka, Phys. Rev. B 28, 6183 (1983).

[3] W. Cable, L. David, R. Parra, Phys. Rev. B 16, 1132 (1977).

[4] A. Arrott, H. Sato, Phys. Rev. 114, 1420, 1427 (1959).

[5] M. Shiga, Y. Nakamura, J. Phys. Soc. Jpn. 40, 1295 (1976).

[6] V.L. Moruzzi, P.M. Marcus, Phys. Rev. B 47, 7878 (1993).

[7] M.E. Elzain, A.A. Yousif, Hyperf. Interact. 94, 1873 (1994).

[8] J. Deniszczyk, Acta Phys. Pol. A 97, 583 (2000).

[9] O.K. Andersen, O. Jepsen, Phys. Rev. Lett. 53, 2571 (1984).

[10] V. von Barth, L. Hedin, J. Phys. C, Solid State Phys. 5, 1629 (1972).

[11] C.D. Hu, D.C. Langreth, Phys. Scr. 32, 391 (1985).

[12] A.F. Tatarchenko, N.I. Kulikov, Phys. Rev. B 50, 8266 (1994).

[13] I. Vincze, Phys. Status Solidi A 7, K43 (1971). 